\title{
Home testing for COVID-19: lessons from direct to consumer genetics
}

\author{
Theodore E. Wilson ${ }^{1} \cdot$ Colin Halverson ${ }^{2,3}$
}

Received: 12 May 2020 / Accepted: 1 June 2020 / Published online: 16 June 2020

(C) Springer-Verlag GmbH Germany, part of Springer Nature 2020

\begin{abstract}
On March 11th, 2020, COVID-19 was declared a worldwide pandemic. Publicly available testing has lagged, and tech entrepreneurs have quickly volunteered to fill this gap. Over the last two decades, genetic testing ordered outside of a clinic and without the involvement of a physician has been a way for the average individual to get genetic testing. In this commentary, we discuss the lessons learned from this parallel case from genetics and suggest regulatory caution in establishing direct-to-consumer COVID testing.
\end{abstract}

On March 11th, 2020, the World Health Organization officially declared the coronavirus disease "COVID-19" a worldwide pandemic. At the time of writing, there are nearly 5,000,000 confirmed cases and 325,000 deaths. Between the city of Wuhan, the northern region of Italy, Spain, Germany, and New York City, this virus will at best be characterized by pockets of high morbidity and mortality. To date, the best intervention has been early testing to break the chain of transmission. However, during the first few weeks of this March, early testing in the United States lagged.(Center for Disease Control 2020)

As just one of many examples of the faltering roll-out of diagnostic testing, the United States opted out of receiving a World Health Organization test kit, which had been shipped to over 60 other countries by early February. The United States in particular was slow to provide an alternative to the WHO's kits, and the first batch produced by the Centers for Disease Control (CDC) in February was flawed. By mid-March, the Department of Health's test kits were still limited to select "high-risk" international travelers, or only to patients who were already critically ill. While there is not a published

Theodore E. Wilson

theowils@iu.edu

1 Department of Medical and Molecular Genetics, Indiana University School of Medicine, 975 W. Walnut St., Indianapolis, IN 46202, USA

2 Center for Bioethics, Indiana University, Indianapolis, IN, USA

3 Department of Anthropology, Indiana University, Bloomington, IN, USA national inventory of available test kits— government officials having kept such numbers confidential-in the second week of March, in our own state of Indiana, we only had 40 kits available to test a population of 6.5 million. Well into March, published values from the United States CDC reported only several thousand specimens tested in total.(Center for Disease Control 2020)

As an example of the discordance between testing and number of cases, retrospective serologic studies of New York City released on May 2nd representing spread through the month of March found that up to $12.3 \%$ of a state with 8.4 million residents had recovered from the virus.(Cuomo 2020) Testing using RT-PCR from a comparable time found only 67,000 total cases (COVID-19 Tracking Project).

Tech entrepreneurs have already volunteered to fill this gap in testing need by streamlining how individuals can receive RT-PCR testing for COVID-19 in many ways. For one innocuous example, the pharmacy conglomerate CVS is offering limited drive-through testing without a prescription to individuals who self-screen as positive per CDC criteria. Other, more creative solutions include those by Amazon and the Gates Foundation, which propose a system where all individuals could receive screening through an online portal, order a test kit through the mail, complete testing at home, and then receive results with instructions on whether and how to selfquarantine.(Farr 2020) This may sound surprising, but the current Amazon marketplace already advertises and sells a variety of the following: paternity, ancestry, allergy antibody, gut microbiome, colon cancer, and cholesterol home test kits, all without the restriction of an ordering healthcare provider. Guidance is instead offered by a "Which DNA test kit should you get" Amazon-sanctioned editorial, which discusses customer service and turn-around time. 
This methodology — which shortcuts the usual healthcare model of an appointment with a primary care clinician, history and physical examination, prescription for testing, and followup counseling by phone or in clinic - is considered a primary feature and not a flaw of a streamlined approach to COVID-19 testing. Evaluating this sort of testing modality is particularly challenging, because evidence for or against this kind of expanded access will only become available slowly. Comparative analysis can help us understand what benefits and risks might exist for direct-to-consumer (DTC) COVID19 testing and can be used to generate hypotheses that would need follow-up research for those individuals who seek out such testing. As we move forward with home testing, it is worth remembering that there are lessons to learn from other at-home testing methodologies. Thus, we suggest a comparison of such COVID-19 testing with direct-to-consumer genetics.

DTC genetic testing is ordered outside of a clinic and without the involvement of a physician. By and large, it has been a way for the average individual to get genetic testing for a variety of reasons, including to receive non-clinical knowledge about ancestry as well as alarming and medically actionable knowledge about pathogenic variants that impute a high risk of cancer. While such DTC testing has undoubtedly increased access, it has grown to become a parallel bioinformatics market in addition to - but markedly separate fromclinical services.

Unfortunately, DTC genetics has yet to live up to its promise. Even after being in use for over two decades, there are still significant clinical and ethical issues with false positives, false negatives, outlandish interpretations, privacy, and lack of preand post-test counseling.(Frueh et al. 2011; Kutz 2010; Stewart et al. 2018; Tandy-Connor et al. 2018) What is perhaps most concerning is that these criticisms have been ongoing, and issues of privilege and privacy have been identified as ethical shortcomings since its inception. What is particularly striking is that DTC genetic testing has not managed to respond fully to these criticisms since they were first levied many years ago. While some less conscientious DTC genetics labs may exist, sound advice to patients considering DTC genetics would still be, "Caveat emptor!"

While features like increased access may underscore the beneficent impulse underlying such DTC endeavors, as in the role of the pharmaceutical industry,(Sunder 2006) this does not preclude the concomitant emergence of ethical quandaries introduced by this novel delivery model. These actions avoid serious moral scrutiny and instead are described as "philanthropic," characterized as beneficent by the mass media and other public discourse. But there are other ethical principles to consider, some of which-like justice - may in fact be at odds with a calculus of pure beneficence. What mechanisms of access will guarantee a just distribution of test kits if this process is overseen by neither the government nor the medical system? We must ask ourselves the following: What are the democratic trade-offs in private versus public authority in these domains? What checks and balances will control the private sector's private interests? How do we incentivize public entities to maintain their role in the provision of such care when private entities enter the arena. Likewise, with important data on COVID-19 sequestered from the public domain, who will conduct follow-up studies on the testing that has occurred.

DTC genetics has not proved an equalizing force that democratizes access to genomic information. Rather, it has emerged as a novelty available to consumers who currently have the resources to expend on it. We have already watched while numerous celebrities have received early COVID-19 testing, raising concerns that financial means and status allow individuals to utilize a scarce resource unjustly. Moreover, results from DTC testing are often not reliable enough to be treated as actionable, and even when they are, it is unclear who might be liable were these outside results to be used to prescribe a treatment or intervention.(Frueh et al. 2011) If such a DTC scenario manifests for COVID-19, a well-intentioned, at-home test for individuals who are worried that they are infected would offer little more than the generalized advice published by the WHO, viz. to "Stay home if you feel unwell." It is very plausible that DTC COVID-19 testing will exacerbate the strain on US healthcare rather than provide necessary relief.

As the world moves forward with COVID-19 testing using any means possible - including a DTC approach — this comparative analysis can generate further studies into what potential downsides might exist for tests obtained on-demand. It is true that we need all the help we can get, and every sector will need to muster resources to fight this pandemic and temper its associated severe economic hardships. If a DTC method is to be used, though, it must be done in a manner that provides relief to the system instead of only to overanxious and economically privileged individuals or regions. Test results need to be integrated into existing healthcare medical records, and they must be produced in a way that ensures that they are reliable enough to make sound clinical decisions.

Acknowledgments Special thanks to the many health care workers at the front line of this pandemic who are already tirelessly providing extraordinary and necessary services. The authors of this manuscript have no financial conflicts of interest and no affiliations or speaking agreements with any diagnostic laboratories.

\section{Compliance and ethical standards}

Conflict of interest Theodore Wilson declares that he has no conflict of interest. Colin Halverson declares that he has no conflict of interest. This article does not contain any studies with human participants or animals performed by any of the authors. 


\section{References}

Center for Disease Control (2020) Coronovirus Disease 2019 (COVID19): Testing in U.S. Accessed March 20, 2020. https://www.cdc. gov/coronavirus/2019-ncov/cases-updates/testing-in-us.html.

Cuomo AM (2020) Amid Ongoing COVID-19 Pandemic, Governor Cuomo Announces Results of Completed Antibody Testing Study of 15,000 People Showing 12.3 Percent of Population Has COVID19 Antibodies. New York State: Information on Novel Coronavirus. Accessed March 20, 2020. https://www.governor.ny.gov/news/ amid-ongoing-covid-19-pandemic-governor-cuomo-announcesresults-completed-antibody-testing

Farr C (2020) Amazon and Gates Foundation may team up to deliver coronavirus test kits to Seattle homes. CNBC. Accessed March 20, 2020. https:/www.cnbc.com/2020/03/11/amazon-care-gatesfoundation-may-deliver-coronavirus-tests-in-seattle.html

Frueh FW, Greely HT, Green RC, Hogarth S, Siegel S (2011) The future of direct-to-consumer clinical genetic tests. Nat Rev Genet 12(7): 511-515. https://doi.org/10.1038/nrg3026
Kutz G (2010) Direct-To-Consumer Genetic Tests: Misleading Test Results Are Further Complicated by Deceptive Marketing and Other Questionable Practices. General Accounting Office.

Stewart KFJ, Wesselius A, Schreurs MAC, Schols AMWJ, Zeegers MP (2018) Behavioural changes, sharing behaviour and psychological responses after receiving direct-to-consumer genetic test results: a systematic review and meta-analysis. J Community Genet 9(1):118. https://doi.org/10.1007/s12687-017-0310-z

Sunder Rajan K (2006) Biocapital: The Constitution of Postgenomic Life. Duke University Press

Tandy-Connor S, Guiltinan J, Krempely K et al (2018) False-positive results released by direct-to-consumer genetic tests highlight the importance of clinical confirmation testing for appropriate patient care. Genet Med 20(12):1515-1521. https://doi.org/10.1038/gim. 2018.38

Publisher's note Springer Nature remains neutral with regard to jurisdictional claims in published maps and institutional affiliations. 